\title{
Metrics Visualization Techniques Based on Historical Origins and Functional Layers for Developments by Multiple Organizations
}

\author{
Ryosuke Ishizue*, Hironori Washizaki ${ }^{\dagger}$ and Yoshiaki Fukazawa* \\ Washizaki Laboratory, Department of Computer Science and Engineering \\ Waseda University, 3-4-1 Ohkubo, Shinjuku-ku \\ Tokyo 169-8555, Japan \\ *ishizue@ruri.waseda.jp \\ ${ }^{\dagger}$ washizaki@waseda.jp \\ \$fukazawa@waseda.jp \\ Sakae Inoue§, Yoshiiku Hanai ", Masanobu Kanazawa" \\ and Katsushi Namba** \\ Fujitsu Connected Technologies Limited \\ 4-1-1 Kamikodanaka, Nakahara-ku, Kawasaki-shi \\ Kanagawa 211-8588, Japan \\ \$inoue.sakae@jp.fujitsu.com \\ 『hanai.yoshiiku@jp.fujitsu.com \\ "kanazawa.masano@jp.fujitsu.com \\ **nanba@jp.fujitsu.com \\ Received 30 January 2017 \\ Revised 25 July 2017 \\ Accepted 26 October 2017 \\ Published 23 November 2017
}

Software developments involving multiple organizations such as Open Source Software (OSS)based projects tend to have numerous defects when one organization develops and another organization edits the program source code files. Developments with complex file creation, modification history (origin), and software architecture (functional layer) are increasing in OSSbased development. As an example, we focus on an Android smart phone and a VirtualBox development project, and propose new visualization techniques for product metrics based on file origin and functional layers. One is the Metrics Area Figure, which can express duplication of edits by multiple organizations intuitively using overlapping figures. The other is Origin City, which was inspired by Code City. It can represent the scale and other measurements, while simultaneously stacking functional layers as 3D buildings. The contributions of our paper are to propose new techniques, implement them as web applications, and share the results of our questionnaire. Our proposed techniques are useful not only to visualize the measured metrics, but also to improve the product quality.

Keywords: Metrics; origin; functional layer; visualization.

This is an Open Access article published by World Scientific Publishing Company. It is distributed under the terms of the Creative Commons Attribution 4.0 (CC-BY) License. Further distribution of this work is permitted, provided the original work is properly cited. 


\section{Introduction}

Software projects developed by multiple groups or organizations have more complex source file creations, modification histories, and software architectures. ${ }^{\text {a }}$ This complexity can lead to issues in open source software (OSS)-based software developments. Previous studies focusing on OSS-based software developments have tackled problems with various approaches $[2,3]$. Herein, we focus on Android smart phone developments, which often incur problems. Platformer provides the base of Android as an OSS. Some developers use Platformer to create their own products. In a development involving multiple organizations, it is important to focus on which organization created which program source code file because files edited by multiple organizations tend to have more defects than ones edited by a single organization [4]. Additionally, it is important to know which layer of the architecture that a file belongs (e.g. Kernel, Driver, Framework, Application, etc.). One merit of a layered architecture is that the dependencies remain in a local layer. However, one demerit is that a severe problem can occur when the behavior of a layer changes (Cascades of changing behavior) [5]. Problems occurring in lower layers can spread to higher layers. Moreover, identifying the origin of a defect in a lower layer is difficult. Thus, it is very dangerous to develop software without knowing which layer a file belongs.

Developers tend to have difficulty reviewing products because adequate methods to effectively consider these two properties do not exist [1-3]. This is our motivation. This study proposes a new method to address this issue. Our goal is to improve the product quality of Android smart phone software.

First, the terms used in this paper are defined.

Definition 1 - Origin: The creation and modification history of a file. This term was defined in a previous study [1, 4].

Definition 2 - Functional layer: Classification of software structure by function. The basic architecture of software that our study focuses on is the layer architecture [5]. If the functional layers have different origins, then development becomes more challenging. Hence, knowing the origin of a file and its functional layers is useful to avoid defects. Moreover, this information leads to improved product quality as product metrics are based on the origin and functional layers.

As a motivating example, our project involving Android smart phone development has three development organizations (Dev.): Platformer (1st Dev.), Chipset Vendor (2nd Dev.), and Fujitsu Connected Technologies (FCNT) (3rd Dev.) (Fig. 1).

\footnotetext{
a This paper is an extended version of a paper "Metrics visualization technique based on the origins and function layers for OSS-based development" [1] presented at the fourth IEEE Working Conference on Software Visualization (VISSOFT 2016). In the previous paper, we only defined and explained our visualization methods via a small experiment. In this paper, we add extensive functions to the proposed visualization techniques, implement these techniques as a tool, employ them as a use case scenario, conduct another experiment designed for a real development project, and conduct statistical tests of our experiment results. In summary, this paper demonstrates the applicability of our method to real software development projects.
} 


\section{Chipset Vendor(2)}

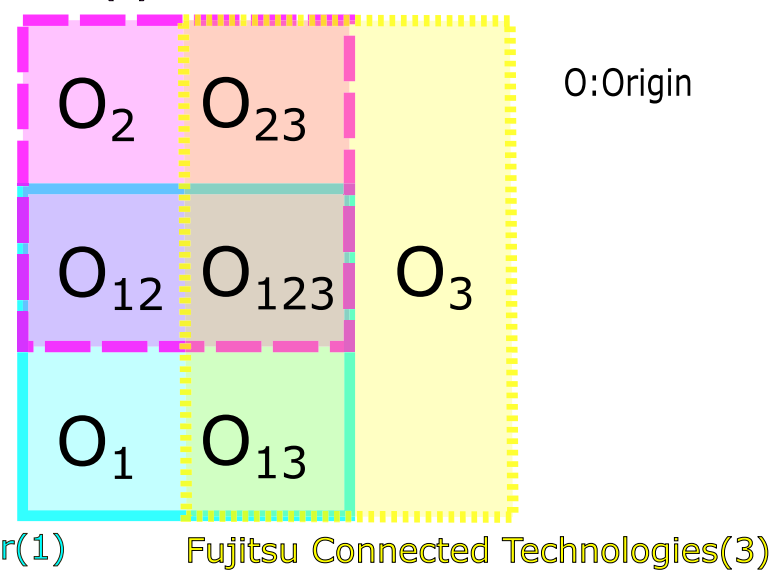

Fig. 1. Origins in our Android smart phone project.

The 1st Dev., 2nd Dev., and 3rd Dev. are in order of involvement in the development. The concept of an origin cannot be applied when an earlier (smaller numbered) organization edits a file of a later (larger numbered) organization. For example, the 1st Dev. cannot edit the files of the 2 nd or $3 \mathrm{rd}$ Dev.

$\mathrm{O}_{1}, \mathrm{O}_{2}$ and $\mathrm{O}_{3}$ denote a file created by Platformer, Chipset Vendor, and FCNT, respectively, whereas $O_{12}$ represents a file created by Platformer and edited by Chipset Vendor. However, Platformer would not edit a file created by Chipset Vendor and FCNT. In our method, a modification history such as $O_{31}$ is not defined and cannot be applied to such a development.

We use the seven functional layers based on the Android Architecture [6]: (1) Linux Kernel, (2) Library, (3) Android Runtime, (4) Library (external OSS), (5) Application Framework, (6) Applications, (7) Others.

When reviewing software, the metrics of each file are extremely difficult to understand using tables, bar charts, bar graphs, or other primitive methods, making it hard to analyze the origin and functional layer. Previous studies have tackled this problem $[7,8]$. For example, it is easy to find the origin of the largest metric with a simple table, but it is challenging to determine the percentage of the total value of the organization (e.g. Total of Platformer $=O_{1}+O_{12}+O_{13}+O_{123}$ ). In another example, Chipset Vendor edited the files that originated from Platformer. Moreover, adding a functional layer further complicates the origin. To help address these issues, we propose a visualization method called the Metrics Area Figure (MAF), which shows the measured metrics of each origin using overlapping circles, rectangles, etc.

Many previous studies have visualized metrics as 2D or 3D objects. Some even used $3 \mathrm{D}$ software visualization as $3 \mathrm{D}$ visualizations can provide more information than 2D visualizations. An especially famous metrics visualization technique is Code City, which represents the scale and other measurements as 3D buildings in a 
city $[9,10]$. Inspired by Code City, we also propose a 3D visualization method called Origin City $(O C)$. OC shows the measured metrics of each origin as well as the functional layers as color-coded stacks. Since our study is the only analytical method to consider concepts such as origin, MAF and OC should aid developers in reviewing products.

The contributions of our paper are that we propose:

(1) Two new visualization techniques, which are useful to improve the product quality,

(2) MAF, which is useful to indicate the origins in a development, and

(3) OC, which is useful to show the functional layers in a development.

This paper focuses on Android smart phone applications as a motivating example. Software development by Platformer or Chipset Vendor is rarely modified by other developers. However, our method is independent of the actual vendor and vendor modification order. Our projects can be applied when multiple organizations are involved historically. For example, our new visualization techniques can be applied to VirtualBox and OpenOffice projects. In these developments, the 1st Dev. and 2nd Dev. are not Platformers and Chipset Vendors. The 2nd Dev. simply took over the developments of the 1st Dev. In more familiar examples, developments by students in schools can be considered. MAF and OC can be applied by assuming that the 1st Dev. is the group of students two years ago, the $2 \mathrm{nd} \mathrm{Dev}$. is the group of students one year ago, and the 3rd Dev. is the students in the current year. Moreover, our visualization techniques do not require a specific number of functional layers or the same name as Android. Although this study uses an Android smartphone development due to the popularity of Android, it is not necessary in our method.

\section{Visualization Method}

\subsection{Visualization procedure}

We implemented two visualization techniques with canvas of HTML5 and JavaScript. Figure 2 overviews the system. First, a user prepares the measurement result as a csv file, which contains measured metrics (e.g. defects, number of public methods/fields, and global variables). For visualization, these metrics must be measurable by a static analysis tool such as Understand as well as contain a functional layer that can be classified by the file path (e.g. kernel/*.* is layer of kernel) and the origin.

To determine the origin of each file in more detail, a directory is prepared until the total number of directories is equal to the number of organizations. The files of each organization are placed into each directory. The diff command is used to find the edited and added files. Metrics of all source code files are measured.

Then, a CSV file summarizing the analysis results (e.g. measured metrics, origins, and layers) are used by the visualization tool. Finally, the results of the visualized data are shown upon pushing the exec button. 


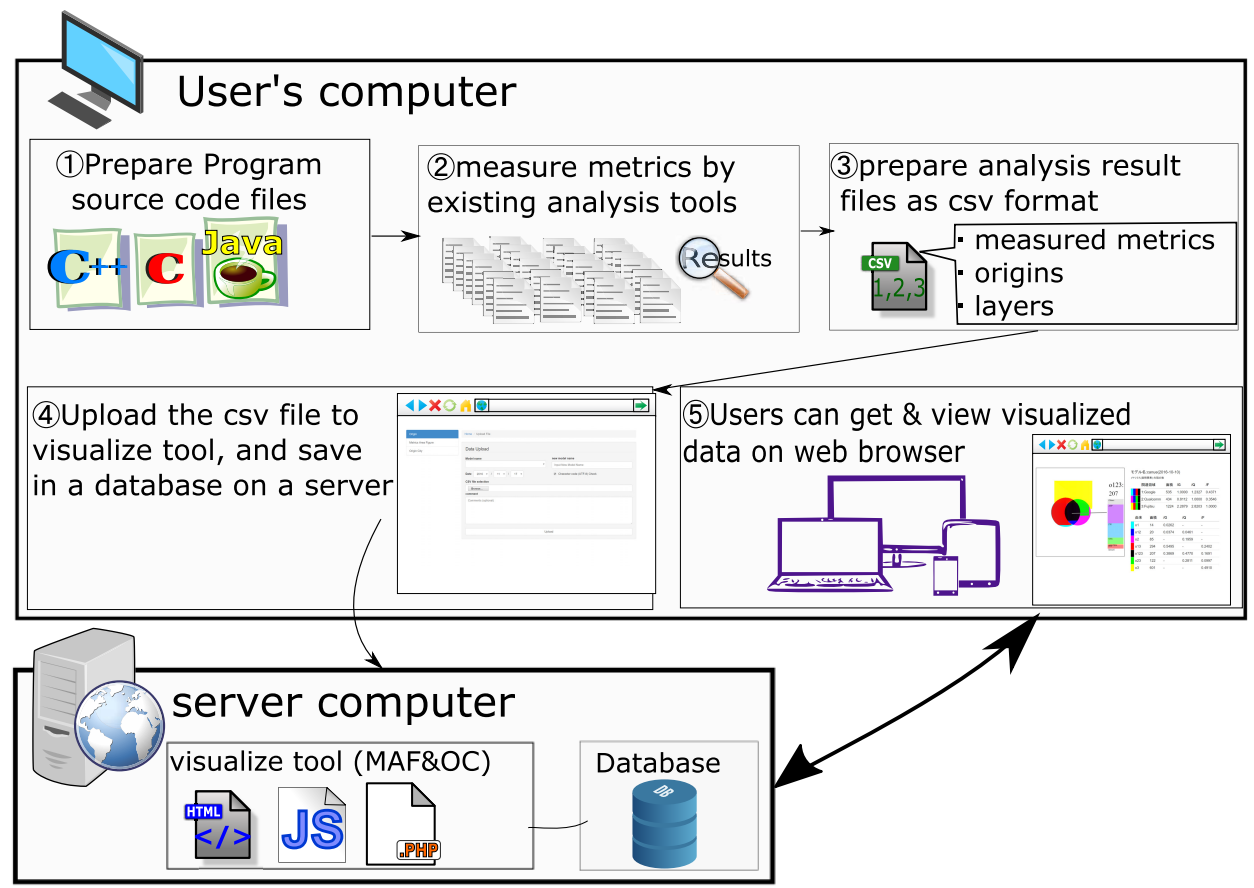

Fig. 2. Overview of the visualization process.

\subsection{Determination of origin}

The procedure to generate MAF and $\mathrm{OC}$ with $N$ organization is as follows. First, there are $2^{N}-1$ regions or buildings in MAF or OC. Each region or building corresponds to the origins represented by $O_{x_{1} \ldots x_{n} \ldots x_{N}}$, where $N$ is the total number of organizations and $n$ is a specific organization. The value of $x_{n}=n$ when the $n$th organization is related to the origin; otherwise the value of $x_{n}=0$, and it is ignored. For example, with five organizations, $O_{124}$ is defined as

$$
O_{x_{1} x_{2} x_{3} x_{4} x_{5}}\left\{N=5, x_{1}=1, x_{2}=2, x_{3}=0, x_{4}=4, x_{5}=0\right\} .
$$

In this example, the 1st organization created a file belonging to this origin. The 2nd and 4th organizations modified the file, but it is not related to the 3rd and 5th organizations. However, our visualizations, especially in MAF, assume that there are several organizations or dozens of origins at most because hundreds or more colored regions may confuse users.

\subsection{Metrics area figure}

MAF has the following features:

(1) A region represents an origin. 
(2) The area of the region is equal to the value of the metric in that origin.

(3) Each area has a unique color based on the organization.

(4) The color represents the origin that includes multiple organizations and is a mixture of each organization's color.

(5) Regions in which the same organizations come into contact with each other form a circle or rectangle, although the shape of each region is not determined.

MAF expresses the duplication of edits by multiple organizations intuitively with overlapping figures. To generate MAF of $N$ organizations, the shape, which has an area equal to the sum of the measured metrics of origins for $x_{1}=1$, is initially painted. Then, another shape, which has an area equal to the sum of the measured metrics of the origins for $x_{2}=2$, is drawn. The overlapping area of the two shapes must equal the sum of the measured metrics of the origins for $x_{1}=1$ and $x_{2}=2$. Next, if $3 \leq N$, the process is repeated while $n \leq N$. The process involves the following steps:

(1) Choose an intersecting point, which is surrounded by most of the regions. The surrounding regions can be up to $O_{x_{1} \ldots x_{(n-1)}}$ (e.g. $n=3, O_{1}, O_{2}, O_{12}$ and outside of figure).

(2) From the intersecting point toward each region whose origin is $O_{x_{1} \ldots x_{(n-1)}}$, new regions, which have areas equal to the sum of the measured metrics of the origins for $x$ equal to the origin of base region plus $x_{n}=n$. (e.g. $n=3, O_{13}$, $\mathrm{O}_{23}, \mathrm{O}_{123}$ and $\mathrm{O}_{3}$ ), are painted.

(3) When all regions, which total $2^{n}-1$, are painted, $n$ is incremented.

Repeating the above process generates MAF of $N$ organizations.

Figure 3 shows an example of an MAF for three organizations. Figure 4 shows the generation process of this example. The cyan shape denotes the sum of the measured

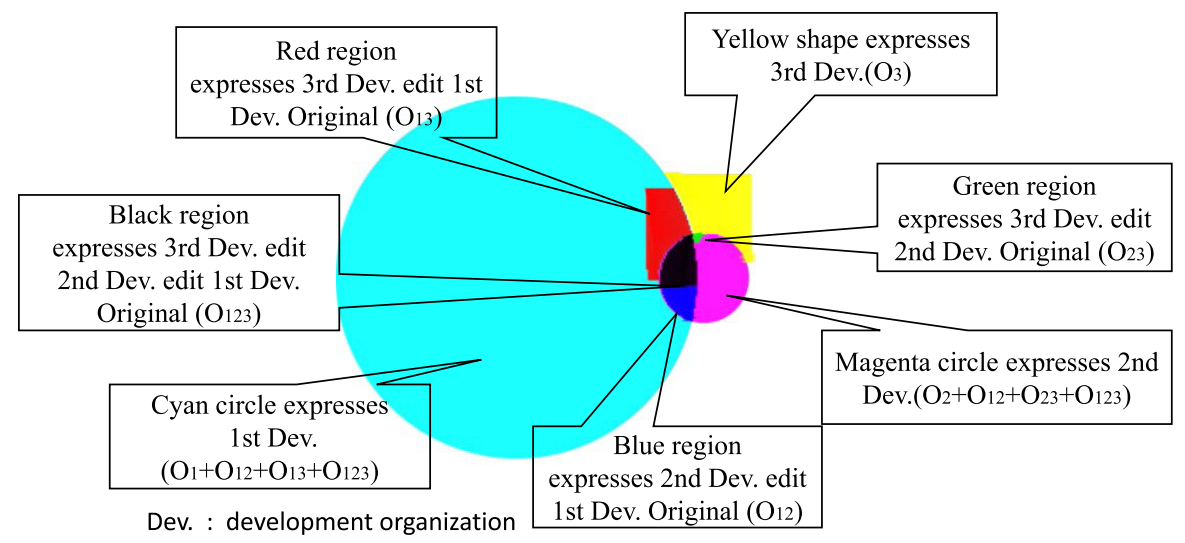

Fig. 3. MAF. 

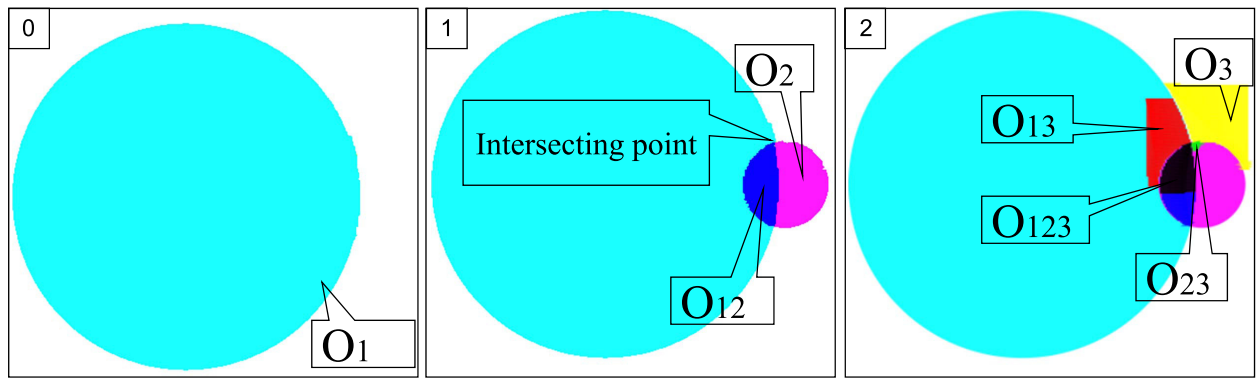

Fig. 4. The MAF generation process of Fig. 3.

metrics of the files created by the 1st Dev. organization $\left(O_{1}\right)$. The magenta shape means the sum of the measured metrics of the files created and edited by the 2 nd Dev. $\left(\mathrm{O}_{2}\right)$, while the yellow shape represents the measured metrics of the files created and edited by the 3rd Dev. $\left(\mathrm{O}_{3}\right)$.

Note that the yellow shape is not a circle because it is mathematically impossible. For example, when $O_{1}=O_{2}=O_{3}=100, O_{123}=50$, and $O_{12}=O_{23}=O_{13}=0$, MAF should draw three overlapping circles whose areas are the same size. If drawn in this manner, $O_{12}$ (blue), $O_{23}$ (green), and $O_{13}$ (red) would have an area larger than 0 (Fig. 5). This is a contradiction. Consequently, the shape of each figure representing each origin is not defined. In most cases, the visualization uses circles and rectangles.

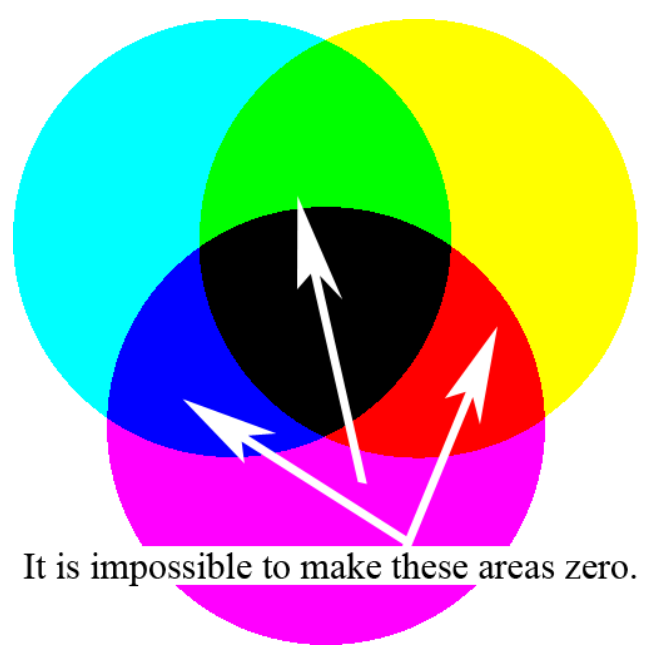

Fig. 5. Examples of mathematical contradiction of MAF.

To generate this figure, a cyan circle $\left(O_{1}\right)$ is painted initially. Then, a magenta circle $\left(O_{2}\right)$ is developed. The overlapping area is painted in blue $\left(O_{12}\right)$. Next, from 
one of the intersecting points, a yellow shape $\left(O_{3}\right)$ is painted outward. Finally, a red shape $\left(O_{13}\right)$, a green shape $\left(O_{23}\right)$, and a black shape $\left(O_{123}\right)$ are painted toward each region.

\subsection{Origin city}

OC has the following features:

(1) A building represents an origin.

(2) The size of the building's base represents the scale of the origin (using the number of files as the origin).

(3) A building is composed of horizontal layers, where each one represents a functional layer.

(4) Each layer has a unique color based on the functional layer that it represents.

(5) The height of each layer is equal to the value of the metric for that functional layer in the origin.

The most important thing is the building position. First, $N$ buildings whose origins are $O_{1}, O_{2}, \ldots, O_{N}$ are placed on a concentric circle. After that, buildings corresponding to $O_{x_{a} x_{b} \ldots x_{n}}\left(1 \leq a<b<\cdots<n \leq N, x_{a}=a, x_{b}=b, \ldots, x_{n}=n\right)$ are placed on the center of gravity of the figure connecting buildings with $O_{a}, O_{b}, \ldots, O_{n}$. For example, $O_{12}$ is placed in the middle of the line connecting $O_{1}$ and $O_{2}$. $O_{123}$ is placed at the center of gravity of a triangle connecting $O_{1}, O_{2}$, and $O_{13} . O_{1234}$ is placed on the center of gravity of a rectangle connecting $\mathrm{O}_{1}, \mathrm{O}_{2}, \mathrm{O}_{3}$, and $\mathrm{O}_{4}$. After all the positions are determined, the buildings are drawn from the center. The radius from the center of the buildings is decided to avoid intersecting each other toward the outside. By repeating the above process, OC of $N$ organizations is generated.

Figure 6 shows an example of $\mathrm{OC}$ with three organizations. The center building corresponds to $O_{123}$, and it is surrounded by buildings $O_{1}, O_{2}$, and $O_{3}$. The $O_{12}$

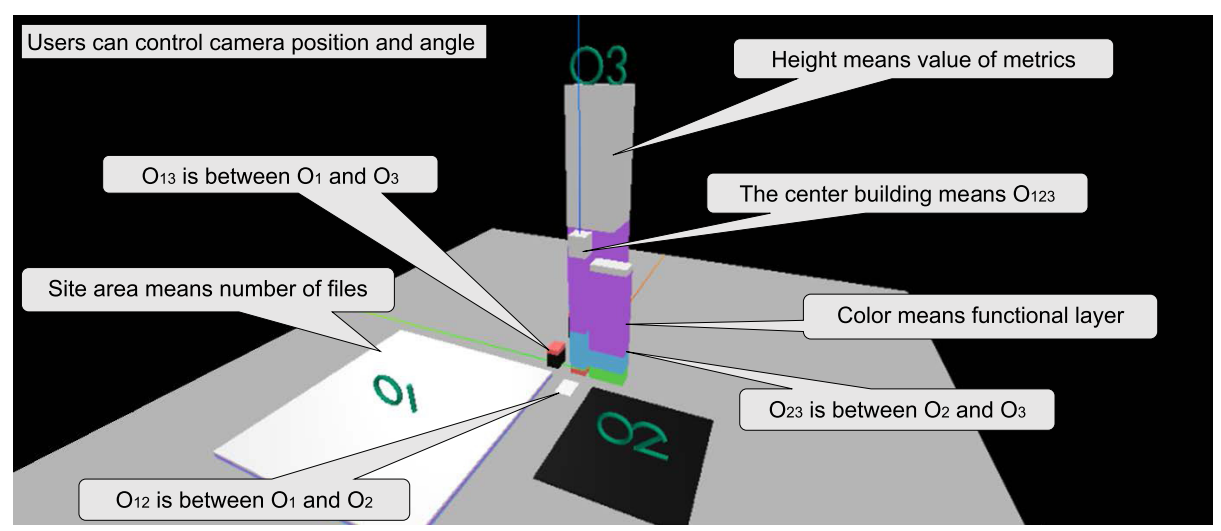

Fig. 6. OC. 
building is between the $O_{1}$ and the $O_{2}$ buildings. The buildings of $O_{13}$ and $O_{23}$ are similarly placed.

\section{Experiment}

We verified the effectiveness of our techniques using Android smart phone projects. As mentioned in the introduction, Android is a large software. The structure of its origin/function layers are difficult to review. In this experiment, we use Android as a typical example.

\subsection{Target and visualization results}

We speculated that our techniques are useful for visualizing a situation at a particular stage of development or comparing stages as a development process progresses. In addition, a comparison of such stages is necessary for managers or developers. Hence, we applied our visualization techniques to Android smart phone projects to verify the effectiveness of these techniques. In this example, there are three organizations:

- 1st Dev. is Platformer.

- 2nd Dev. is Chipset Vendor.

- 3rd Dev. is FCNT.

\subsubsection{Example of metrics area figure}

Figure 7 is an example of MAF, which shows the number of defects. The large yellow region shows that files created by FCNT contain numerous defects. Moreover, the cyan and magenta circles almost completely overlap, demonstrating that the defects are mainly due to the FCNT edit. Such analysis makes it easy to intuitively grasp the origins with many defects. Consequently, developers know to carefully edit such files.

However, MAF cannot distinguish the degree of contribution of the measured metrics by organization. For example, the files of $O_{13}$ may contain many defects prior to editing by FCNT. However, the visualization requires data not edited by Chipset Vendor, data not edited by FCNT, and data edited by FCNT. Thus, user can analyze the degree of contribution in more detail based on all the data and the trend of measured metrics by MAF.

Figure 8 shows additional examples of MAF. They show the results of visualizing a series of data for an Android smart phone project involving the lines of code (LOC). The right example visualizes newer data than the left. The magenta circle on the right is larger than that on the left because the Android version is updated on the right. Moreover, the overlapping area between Platformer and Chipset Vendor $\left(O_{12}\right.$ and $\left.O_{123}\right)$ is larger on the right than on the left. However, the size of the cyan circle is about the same. Thus, MAF helps developers review previous projects. 

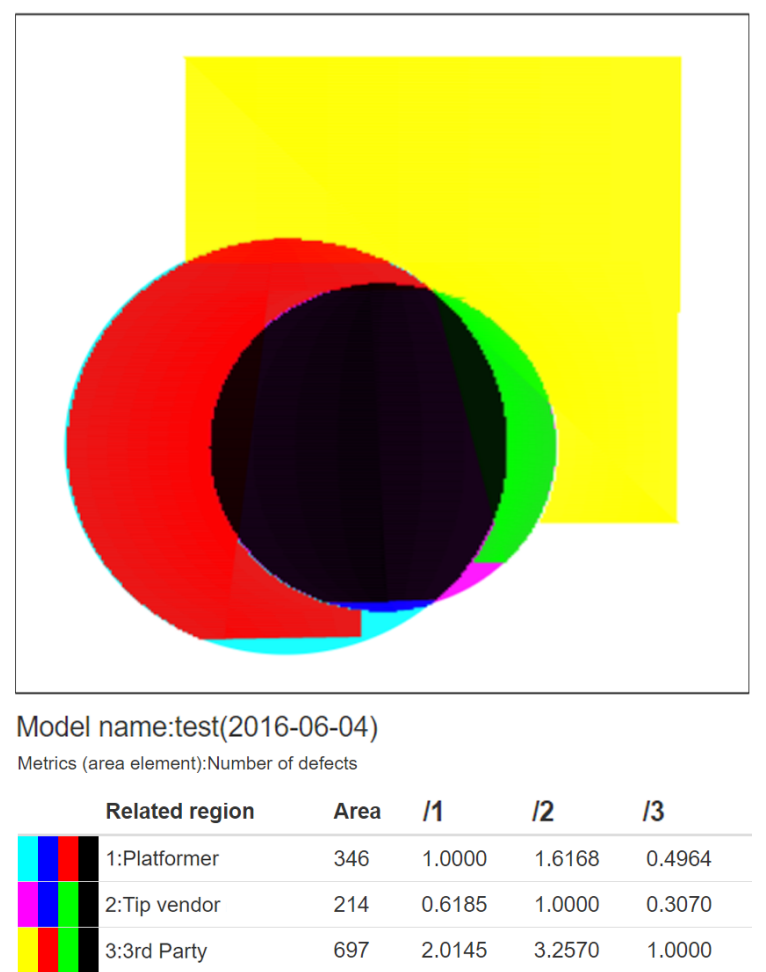

\begin{tabular}{lllll} 
Origin & Area & I & I2 & I3 \\
\hline 01 & 8 & 0.0231 & - & - \\
\hline 012 & 2 & 0.0058 & 0.0093 & - \\
\hline 02 & 2 & - & 0.0093 & - \\
\hline 013 & 153 & 0.4422 & - & 0.2195 \\
0123 & 183 & 0.5289 & 0.8551 & 0.2626 \\
023 & 27 & - & 0.1262 & 0.0387 \\
03 & 334 & - & - & 0.4792
\end{tabular}

Fig. 7. Example of MAF.

By definition, MAF requires a table similar to that in Fig. 7, which shows the measured metrics represented by each area where origins are represented by unique colors. Thus, developers should use MAF as additional material to such tables. If the developer uses only MAF without such tables or knowledge of the definition, it may confuse the developers. In Fig. 3, for example, the red region expresses the 3rd Dev.'s edit of the 1st Dev. $\left(O_{13}\right)$. However, the color indication can be interpreted as the 1st Dev.' edit of the 3rd Dev. This situation is unrealistic due to the definition of Origin and MAF. In such cases, the developer should not apply MAF. 


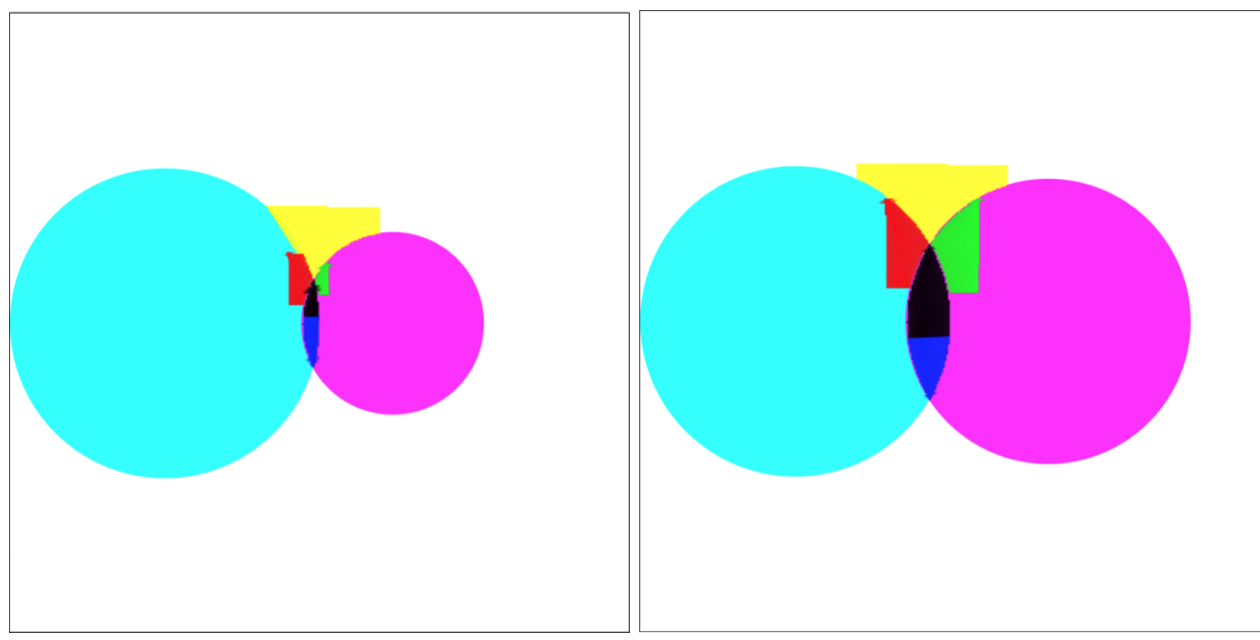

Fig. 8. Example of MAF in an old (left) and a new (right) model.

\subsubsection{Example of origin city}

Figure 9 provides an example of $\mathrm{OC}$ where the number of defects is given as measured metrics. Platformer $\left(O_{1}\right)$ and Chipset Vendor $\left(O_{2}\right)$ created many more files (site areas) than FCNT. Moreover, the site area of $O_{123}$ is the same as $O_{13}$, but its height is much higher, indicating that the ratio of defects is greater in $O_{123}$ than in $O_{13}$. The building color of $O_{13}$ is black and cyan, whereas that of $O_{123}$ is purple and yellow, indicating that the defects in $O_{13}$ are in the lower layers (Kernel and HW Library), whereas those in $O_{123}$ are in the higher layers (APP and FW).

By such an analysis, it is easier to grasp intuitively the origin and functional layer with many defects. As a result, developers are aware to carefully edit such files.

Figures 10 and 11 show additional examples of OC using the same data as the MAF example. The building of $\mathrm{O}_{2}$ in the new model is larger and higher than that in the old model because the Android version has been updated. Moreover, the black $O_{1}$ layer in the new model almost disappears compared to the large black $\mathrm{O}_{2}$ layer in the old model. Consequently, OC can help developers review and analyze previous projects.

\subsubsection{Visualization tool}

We implemented MAF and OC as web application tools. Users can view visualized data after uploading the csv file containing the measured metrics to the tool using a server computer. For example, Fig. 12 shows part of the MAF page. Users can view the visualized data as MAF after selecting the parameters (target data, group, and metrics) from the form at the top of the page. In our web application integrated with 


\section{Model name:test(2016-06-04)}

Metrics:Number of defects

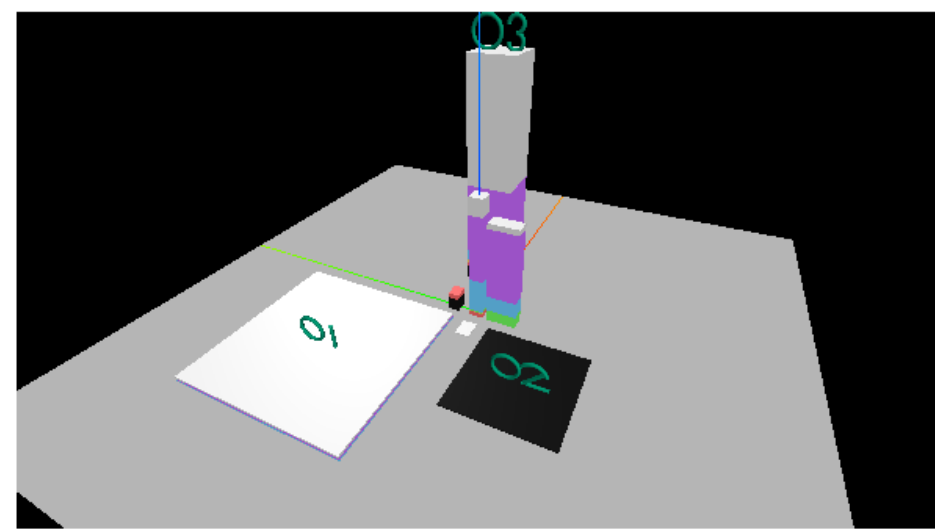

\begin{tabular}{|lrrrrrrrr}
\hline \multicolumn{1}{|c}{ Function layer } & 01 & 012 & 02 & 013 & 0123 & 023 & o3 & Total value \\
\hline Application (APP) & 3 & 0 & 0 & 104 & 97 & 0 & 95 & 299 \\
\hline Application framework (FW) & 2 & 0 & 0 & 24 & 51 & 0 & 17 & 94 \\
\hline Library (external OSS) & 0 & 0 & 0 & 17 & 2 & 0 & 2 & 21 \\
\hline Android Runtinme(SYSTEM) & 0 & 0 & 0 & 0 & 1 & 0 & 3 & 4 \\
\hline HW Library & 0 & 0 & 0 & 0 & 7 & 7 & 7 & 21 \\
\hline Kernel & 0 & 0 & 2 & 0 & 0 & 20 & 55 & 77 \\
\hline Others & 3 & 2 & 0 & 8 & 25 & 0 & 155 & 193 \\
\hline Metrics total value & 8 & 2 & 2 & 153 & 183 & 27 & 334 & 709 \\
\hline Total number of files & 81764 & 741 & 27561 & 1200 & 564 & 426 & 4710 & 116966
\end{tabular}

Fig. 9. Example of OC.

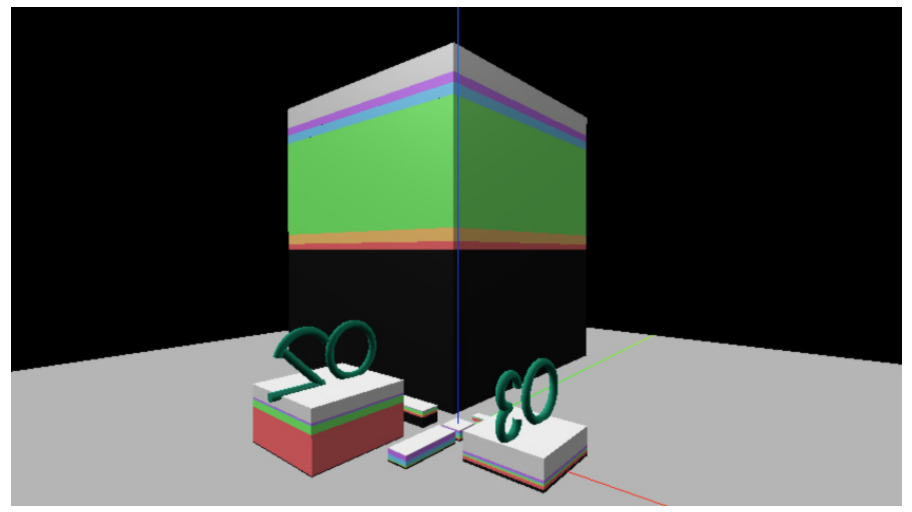

Fig. 10. Example of OC (old model). 


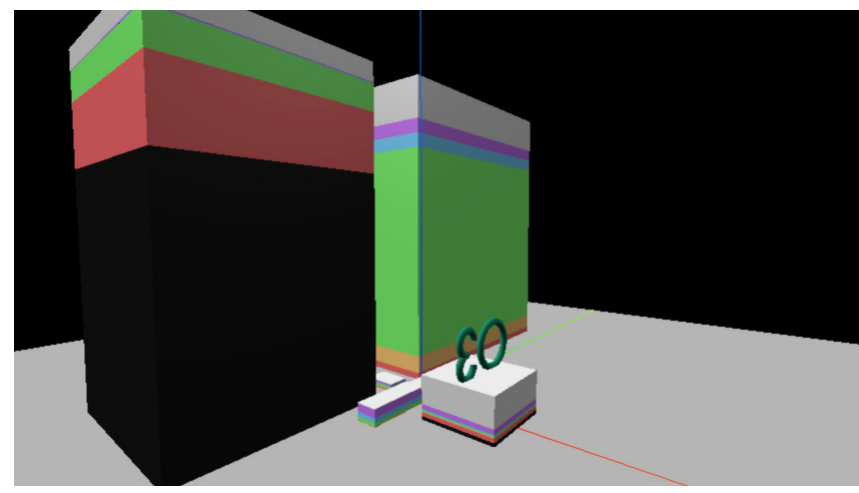

Fig. 11. Example of OC (new model).

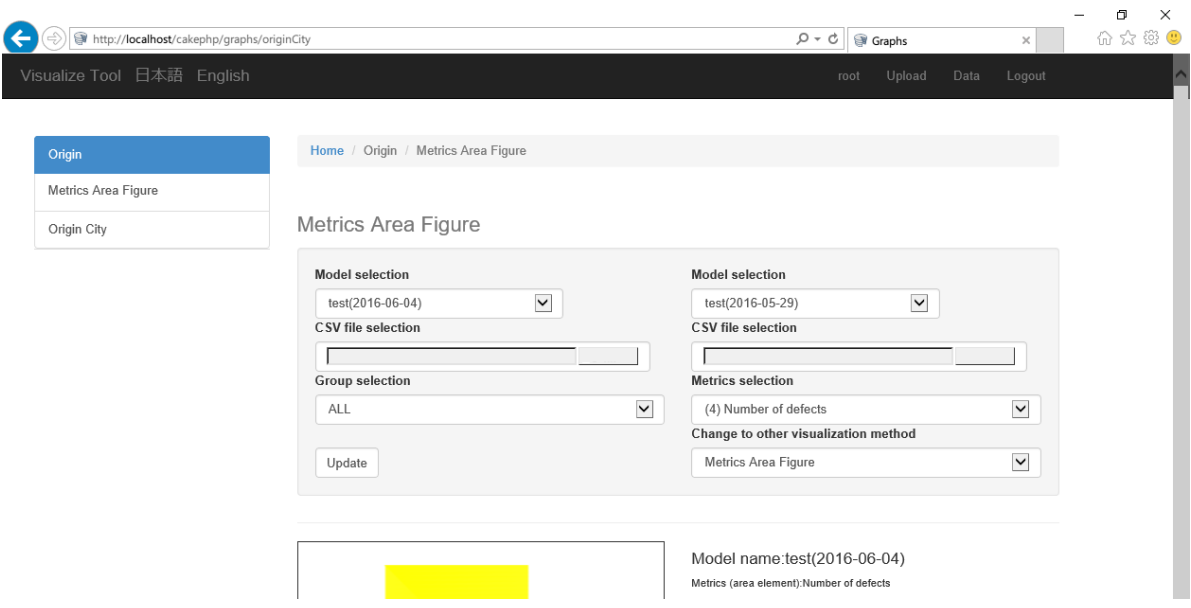

Fig. 12. Page of MAF.

MAF and OC, each technique has basic features as well as two optional features:

(1) A stacked bar chart, which is colored by function layer, is shown by MAF when a user clicks the MAF region. Figure 13 shows an example of this feature, where the height of each layer is equal to the value of the metric for that functional layer in the selected origin. This chart is the same as a cross-section view of the building representing the origin, which is clicked by the user in OC.

(2) Users can switch visualization techniques with the same parameters by selecting another technique and clicking the update button.

Using these features, the following story is conceivable:

(1) First, a user visualizes data as MAF to overview the measured metrics with overlapping areas. 


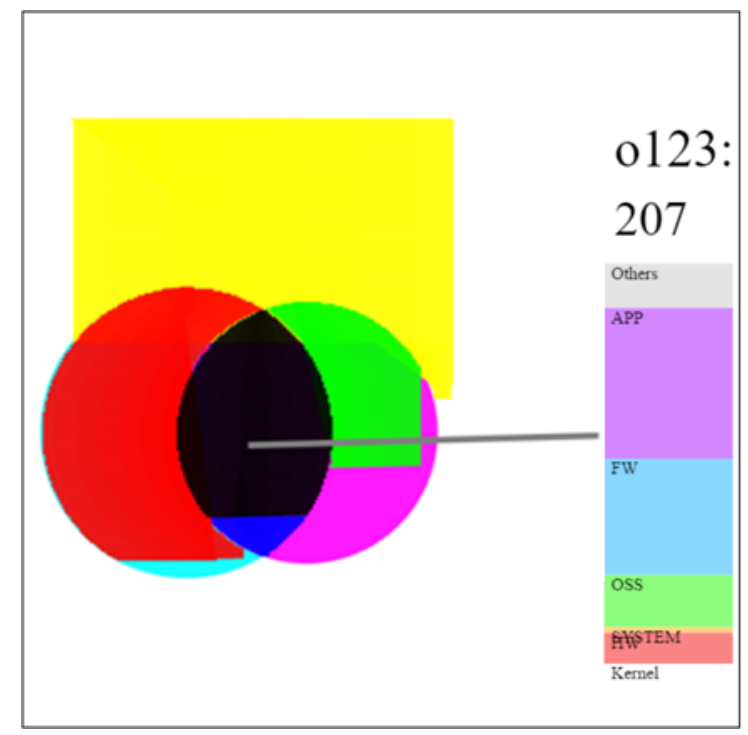

Fig. 13. Example of MAF with layers.

(2) Next, the user clicks the origin of interest to show the stacked bar chart of the layers to see the ratio of each layer by origin.

(3) Then, the user switches the visualization techniques from MAF to OC while maintaining the same parameters to compare all values by layer.

(4) Finally, the user looks at the table at the bottom or side of OC to see detailed values.

It is much easier for developers to analyze the measured metrics based on the origin and the functional layer with MAF and OC. MAF solves problems that are difficult to grasp using the measured metrics of an origin that includes multiple organizations due to overlapping figures. Similarly, the stacks of colored layers in OC address the issue of complex origins and functional layers.

\subsection{Usefulness questionnaire experiment}

\subsubsection{Experiment setting}

We asked developers about the usefulness of our visualization techniques for Android smart phone projects. We speculated that both visualization techniques are useful for senior development personnel like project managers and team leaders to review previous projects and to compare the progress of a current project. We conducted an experiment to verify this hypothesis. This work investigated the following research questions:

- RQ1: Does MAF increase awareness of origins in a development? 
- RQ2: Does OC increase awareness of functional layers in a development?

- RQ3: Are our new visualization techniques useful to improve product quality?

- RQ4: What is the purpose of our new visualization techniques?

- RQ5: Who would find our visualization techniques useful?

To answer these research questions, we implemented a questionnaire about our new visualization techniques.

Table 1 shows 13 questions about the awareness of metrics, origins, and functional layers as well as the 4 questions about which method provides the best visualization. Q1 to Q13 are evaluated on a four-level scale: Very much, Somewhat, A little, and Not at all. Q14 to Q17 are also answered on a four-point scale: Table, Pie/Bar Chart, $\mathrm{MAF}$, and OC.

Table 1. Questions.

\begin{tabular}{cl}
\hline Q & \multicolumn{1}{c}{ Question sentence } \\
\hline 1 & Are you usually aware of the origin? \\
2 & Are you usually aware of the metrics? \\
3 & Are you usually aware of the metrics for each origin? \\
4 & Are you usually aware of the metrics for each functional layer? \\
5 & Do you feel that MAF is useful for awareness of the origin? \\
6 & Do you feel that MAF is useful for awareness of the metrics? \\
7 & Do you feel that MAF is useful for awareness of the metrics for each origin? \\
8 & Do you feel that OC is useful for awareness of the metrics? \\
9 & Do you feel that OC is useful for awareness of the metrics for each functional layer? \\
10 & Do you feel that MAF is useful to improve product quality? \\
11 & Do you feel that OC is useful to improve product quality? \\
12 & Do you feel that MAF is useful for your work? \\
13 & Do you feel that OC is useful for your work? \\
14 & Which method is the best to grasp the detailed value? \\
15 & Which method is the best to grasp the trend of the value? \\
16 & Which method is the best to grasp the value by functional layer? \\
17 & Which method is the best to grasp the value by origin? \\
\hline
\end{tabular}

We implemented a questionnaire, and 18 people belonging to an Android smart phone development project completed it. They were managers, leaders, and various other project members (designer, reviewer, tester, programmer, etc.).

\subsubsection{Experimental results}

Figures 14-18 show the results of all participants and by participant's role.

\section{RQ1: Does MAF increase awareness of origins in a development?}

Q5 to Q7 indicate that about 70-80\% of the respondents feel our new visualization techniques are useful for improving the awareness of metrics based on the origin. As expected, most feel that MAF is useful to understand the origin by comparing the awareness about the metrics by origin without MAF (Q3) and with MAF (Q7). 


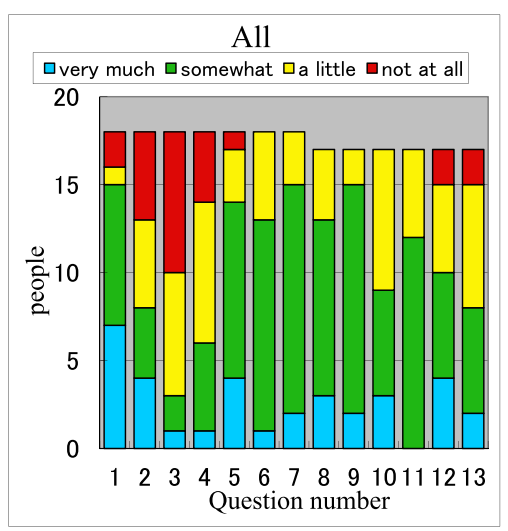

Fig. 14. All responses for Q1 to Q13.

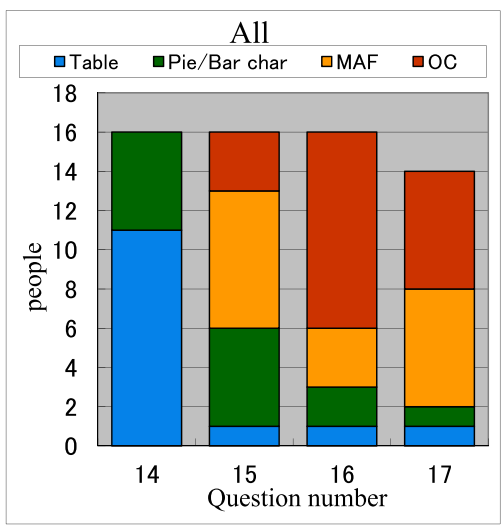

Fig. 15. All responses for Q14 to Q17.

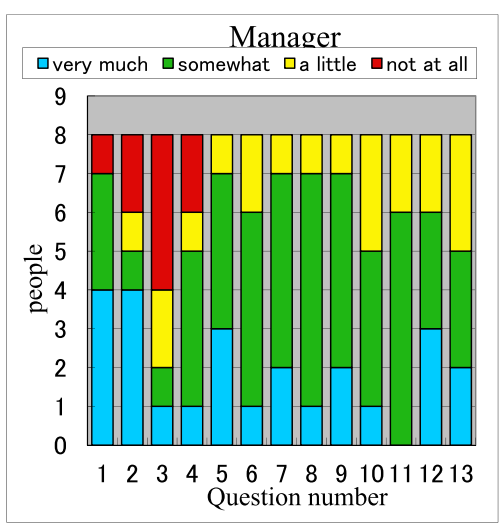

Fig. 16. Managers' responses for Q1 to Q13. 


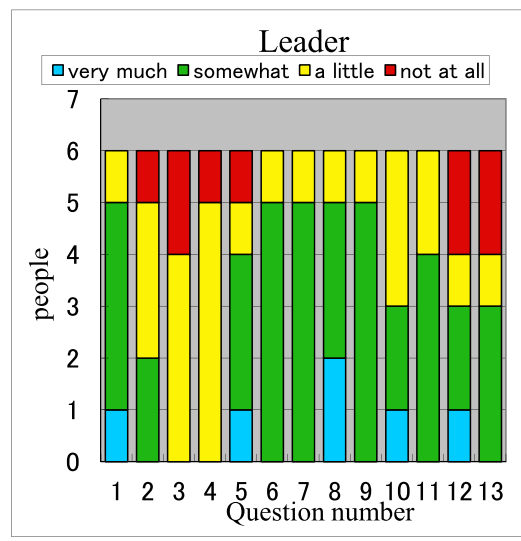

Fig. 17. Leaders' responses for Q1 to Q13.

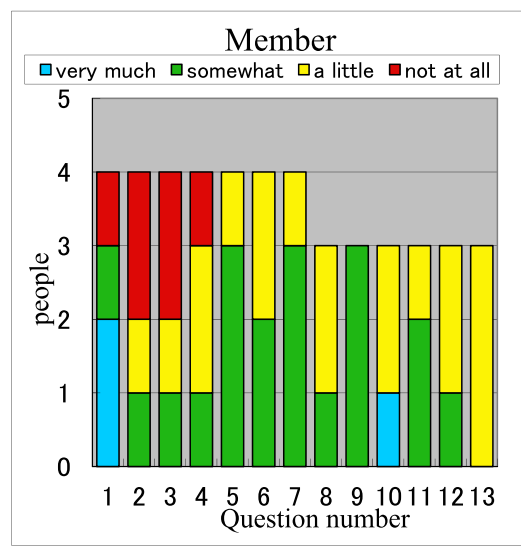

Fig. 18. Members' responses for Q1 to Q13.

\section{RQ2: Does OC increase awareness of functional layers in a development?}

Q4 shows that less than $50 \%$ of developers are aware of the metrics and the functional layers. However, Q8 to Q9 show that about 70-80\% of the people feel our new visualization techniques are useful for improving the awareness of the functional layers. As expected, most feel that OC is useful to understand the functional layers by comparing the awareness about metrics by layer without OC (Q4) and with $\mathrm{OC}(\mathrm{Q} 9)$.

Moreover, Tables 2 and 3 show the analysis of these results for RQ1 and RQ2, including the $p$-values evaluated by a Wilcoxon signed-rank test. These results show a significant difference with or without our techniques with respect to the awareness of the origin and layer. 
Table 2. Analysis of Q3 and Q7 by participant's role. $($ Very $\operatorname{much}=1$, Somewhat $=2$, A little $=3$, Not at all=4).

\begin{tabular}{llllcc}
\hline & \multicolumn{2}{c}{ Median } & & \multicolumn{2}{c}{ Wilcoxon signed-rank test } \\
\cline { 2 - 3 } \cline { 5 - 6 } Roll & Q3 & Q7 & & $p$-value & stat. \\
\hline All & 3 & 2 & & 0.006981 & $<0.01$ \\
Manager & 3.5 & 2 & & 0.095632 & $<0.1$ \\
Leader & 3 & 2 & & 0.053337 & $<0.1$ \\
Member & 3 & 2 & & 0.345779 & n.s \\
\hline
\end{tabular}

Table 3. Analysis of Q4 and Q9 by participant's role. (Very $\operatorname{much}=1$, Somewhat $=2$, A little $=3$, Not at all=4).

\begin{tabular}{llllcc}
\hline & \multicolumn{2}{c}{ Median } & & \multicolumn{2}{c}{ Wilcoxon signed-rank test } \\
\cline { 2 - 3 } \cline { 5 - 6 } Roll & Q4 & Q9 & & $p$-value & stat. \\
\hline All & 3 & 2 & & 0.008771 & $<0.01$ \\
Manager & 2 & 2 & & 0.185877 & n.s \\
Leader & 3 & 2 & & 0.047715 & $<0.05$ \\
Member & 3 & 2 & & 0.345779 & n.s \\
\hline
\end{tabular}

\section{RQ3: Are our new visualization techniques useful to improve product quality?}

Q10 and Q11 confirm that more than half of the participants feel that both methods are useful to improve product quality.

\section{RQ4: What is the purpose of our new visualization techniques?}

According to Fig. 15, tables, pie and bar charts are more useful than our method to grasp the detailed value (Q14), but more people think that MAF is the best method to grasp the trend of a value (Q15). As expected, $63 \%$ of the participants feel that OC is better to grasp the value by functional layers (Q16). Surprisingly, OC is as good of a method to grasp the values of the origins as MAF (Q17). These results indicate that our proposed techniques effectively visualize the measured metrics based on the origin and functional layers.

However, MAF and $\mathrm{OC}$ are not intended to be used without other information such as a table with numeric data. Our proposed visualization approaches are not self-sufficient and self-informative. They require additional information, whereas bar charts and pie charts can be used without any additional information. Thus, these questions (Q14-Q17) are to determine the type of complementary effects when using MAF and OC with other methods.

\section{RQ5: Who would find our visualization techniques useful?}

We hypothesized that the questionnaire results depend on the participant's role. That is, managers would have more positive answers than leaders, while leaders 
Table 4. Analysis of Q1-Q13 by participant's role. (Very much $=1$, Somewhat $=2$, A little $=3$, Not at all=4).

\begin{tabular}{lcccccc}
\hline & \multicolumn{3}{c}{ Median } & & \multicolumn{2}{c}{ Kruskal-Wallis test } \\
\cline { 2 - 3 } Question & Manager & Leader & Member & & $p$-value & stat. \\
\hline 1 & 1.5 & 2 & 1.5 & & 0.639827 & n.s \\
2 & 1.5 & 3 & 3.5 & & 0.263763 & n.s \\
3 & 3.5 & 3 & 3.5 & & 0.998077 & n.s \\
4 & 2 & 3 & 3 & & 0.321359 & n.s \\
5 & 2 & 2 & 2 & & 0.359739 & n.s \\
6 & 2 & 2 & 2.5 & & 0.512458 & n.s \\
7 & 2 & 2 & 2 & & 0.448612 & n.s \\
8 & 2 & 2 & 3 & & 0.185302 & n.s \\
9 & 2 & 2 & 2 & & 0.558035 & n.s \\
10 & 2 & 2.5 & 3 & & 0.927933 & n.s \\
11 & 2 & 2 & 2 & 0.935507 & n.s \\
12 & 2 & 2.5 & 3 & 0.271444 & n.s \\
13 & 2 & 2.5 & 3 & 0.210538 & n.s \\
\hline
\end{tabular}

would have more positive responses than members. We inferred that our methods are more useful for management because managers generally have more management tasks than leaders, who have more management tasks than members. However, according to Table 4, which shows the analysis of this result, including the $p$-values evaluated by the Kruskal-Wallis test, we found that the overall results are positive, but there are no significant differences between the three groups. According to Tables 2 and 3, which also show the analysis by participant's role, the results of the manager and the leader have statistically significant differences, while that of the members are statistically insignificant. Thus, managers have more positive answers than leaders, and leaders have more positive responses than members when we focus on a combination of answers. We speculate that our methods are more useful for participants in managerial positions who are not aware of metrics by origin as our methods increase awareness of each functional layer.

\subsection{Practical review process experiment}

\subsubsection{Experiment setting}

Next, we conducted another experiment assuming a practical review process. We hypothesized that both visualization techniques are useful in the review process to analyze deeply and reduce time. We conducted an experiment to verify these hypotheses.

This work investigated the following research questions:

- RQ6: Do MAF and OC reduce the time for review process?

- RQ7: Do MAF and OC enable deeper analysis? 
We chose VirtualBox as the target project. VirtualBox is an open source project developed by three organizations. In order of development, they are Innotek (1st Dev.), Sun Microsystems (2nd Dev.), and Oracle Corporation (3rd Dev.). We measured and prepared following metrics: (1) Number of files, (2) Number of defects, ${ }^{\text {b }}$ and (3) Cyclomatic complexity by origins and functional layers. We prepared four tasks. Table 5 shows the examination questions for each task.

Table 5. Examination questions.

\begin{tabular}{|c|c|}
\hline $\mathrm{T}$ & Examination sentence \\
\hline 1 & $\begin{array}{l}\text { Simple task with MAF: Compare data on the number of defects on } 2012 / 05 / 31 \text { and } 2017 / 04 / 21 \text {. } \\
\text { Let } \mathrm{S} \text { be the total number of defects in the files }\left(O_{1}, O_{12}, O_{13} \text {, and } O_{123}\right) \text { created by the } 1 \text { st Dev. } \\
\text { Let R be the "number of defects in an origin" } \div \mathrm{S} \text {. Which origin }\left(O_{1}, O_{12}, O_{13} \text {, and } O_{123}\right) \text { has the } \\
\text { greatest growth rate for R? How many times did the number of defects increase? }\end{array}$ \\
\hline 2.1 & $\begin{array}{l}\text { Simple Task with OC: Use data on the number of defects and the number of files on } 2012 / 05 / 31 \text {. } \\
\text { Let } F 3, F 13, F 23 \text {, and } F 123 \text { be the number of files created or edited by the 3rd Dev. } \\
\left(O_{3}, O_{13}, O_{23} \text {, and } O_{123}\right) \text {. Let } D 3, D 13, D 23 \text {, and } D 123 \text { be the number of defects. Which has more } \\
\text { defects relative to the number of files }(D \div F) \text { ? What is the ratio of }(D \div F) \text { ? }\end{array}$ \\
\hline 2.2 & $\begin{array}{l}\text { Simple Task with OC: Compare data for the number of files on } 2012 / 05 / 31 \text { and } 2017 / 04 / 21 \text {. Let } F_{n} \\
\text { be the number of files of } O_{n} \text {. Let } L_{m} F_{n} \text { be the number of files for each layer of } O_{n} \text {. Which origin } \\
\text { shows the layer with the largest increase in the ratio of } L_{m} F_{n} \div F_{n} \text {. What is the ratio of } \\
L_{m} F_{n} \div F_{n} \text { ? }\end{array}$ \\
\hline 3 & $\begin{array}{l}\text { Task with MAF: Describe what you noticed in the data regarding the number of files, number of } \\
\text { defects, and cyclomatic complexity on } 2012 / 05 / 31 \text { and } 2017 / 04 / 21 \text {. (What should developers be } \\
\text { careful about in the future, especially "when comparing the number of files and the number of } \\
\text { defects, and the number of defects increases when the number of files of the } O_{3} \text { has not changed. } \\
\text { It seems that developers tend to make defects in this origin.") }\end{array}$ \\
\hline 4 & $\begin{array}{l}\text { Task with OC: Similar to T3, describe what you noticed in the data about the number of files, } \\
\text { number of defects, and cyclomatic complexity on } 2012 / 05 / 31 \text { and } 2017 / 04 / 21 \text {. }\end{array}$ \\
\hline
\end{tabular}

The 12 participants were undergraduate or graduate students of computer science and engineering. Half undertook the tasks with visualization of MAF and OC, and the half did not use MAF and OC. All participants used the tables with numeric data. Tasks 1 and 2 were simple calculation tasks. Tasks 3 and 4 were description tasks. We measured the response time for each task. We used number of characters for Tasks 3 and 4 instead of correct answer ratio. Additionally, we measured the correct answer ratio for tasks 3 and 4 .

\subsubsection{Experiment results}

\section{RQ6: Do MAF and OC reduce the time for the review process?}

Table 6 shows the time to answer each task: total time of simple calculation tasks (Tasks 1 and 2), total time of description tasks (Tasks 3 and 4), and total time of all tasks. Contrary to our expectations, Table 6 indicates that the group without MAF

\footnotetext{
bWe counted "fix" and "correct" in commit messages as indicators.
} 
Table 6. Response answers [s].

\begin{tabular}{lccrrr}
\hline & \multicolumn{2}{c}{ Mean } & & \multicolumn{2}{c}{ Welch's T test } \\
\cline { 2 - 3 } $\mathrm{T}$ & Without MAF \& OC & With MAF \& OC & & $p$-value & Stat. \\
\hline 1 & 371 & 654 & & 0.070452 & $<0.1$ \\
2 & 838 & 1006 & & 0.659535 & n.s \\
3 & 410 & 468 & & 0.593486 & n.s \\
4 & 341 & 646 & & 0.952179 & n.s \\
$1+2$ & 1210 & 1661 & & 0.350841 & n.s \\
$3+4$ & 750 & 750 & & 0.712718 & n.s \\
$1+2+3+4$ & 1960 & 2475 & & 0.336443 & n.s \\
\hline
\end{tabular}

and $\mathrm{OC}$ solved the tasks faster than the group without MAF and OC, while there is no significant difference between the results of tasks, except for Task 1 . However, the group using the proposed method may require more time because they have more reference materials.

\section{RQ7: Do MAF and OC enable deeper analysis?}

Table 7 shows the number of correct answers (Tasks 1 and 2) and the number of characters of the answer (Tasks 3 and 4). As expected, the correct answer ratio of the group with MAF and OC is higher than the group without MAF and OC, but the difference is insignificant. The group with MAF and OC described more texts than the group without MAF and OC, and the results are statistically significant.

Table 7. Number of correct answers $[\mathrm{s}](\mathrm{T} 1,2)$ and number of characters $(\mathrm{T} 3,4)$.

\begin{tabular}{lccccr}
\hline & \multicolumn{2}{c}{ Mean } & & \multicolumn{2}{c}{ Welch's T test } \\
\cline { 2 - 3 } $\mathrm{T}$ & Without MAF \& OC & With MAF \& OC & & $p$-value & Stat. \\
\hline 1 & 0.83 & 1 & 0.363217 & n.s \\
2 & 1.67 & 1.83 & & 0.5497 & n.s \\
3 & 57.5 & 120.5 & & 0.013353 & $<0.05$ \\
4 & 63.3 & 123.5 & & 0.099616 & $<0.1$ \\
$1+2$ & 2.5 & 2.83 & & 0.2618 & n.s \\
$3+4$ & 120.83 & 244 & & 0.031352 & $<0.05$ \\
\hline
\end{tabular}

\subsection{Threats to validity}

The questionnaire was carried out after our demonstration for developers. The explanation was almost the same as the example in this paper. Therefore, the results of this experiment may change if developers employ our visualization tool in their own development works. This is a threat to the internal validity.

These results are from one group in one company. Some of the participants were already familiar with metrics, origins, and functional layers. If these experiments are repeated with another group or organization unfamiliar with these concepts, the results may differ. This is a threat to the external validity. 


\section{Related Work}

In this section, we describe related works about the origins, Code City, and metrics visualization software, in general.

Sato et al. examined the software in a project involving multiple organizations [4, 11] with an emphasis on the effects of organizational changes on product metrics and defects [4]. They analyzed open source projects to investigate the relationships between the file's creation, modification history, and metrics. Then, they defined a file's origin as its creation and modification history. Our proposed visualization techniques, especially MAF, are based on this concept.

A famous existing technique is the Venn diagram. A Venn diagram visualizes a group using a figure. Figure 1, which provides an overview of the origins, is based on a Venn diagram. MAF is also based on a Venn diagram. When a figure A representing group A overlaps with a figure $\mathrm{B}$ representing group $\mathrm{B}$, the overlapped area represents $\mathrm{AB}$. In this case, $\mathrm{BA}$ has same meaning as $\mathrm{AB}$. For MAF, when a figure representing the 1st Dev. overlaps with another figure representing the 3rd Dev., the overlapped area represents $O_{13}$. Unlike a Venn diagram, the overlapped area does not represent $O_{31}$ because the definition of origin does not allow a developer from an earlier phase to edit a file from a later phase. MAF should not be used if such a development occurs. This is the main difference between a Venn diagram and MAF. Moreover, MAF requires a unique color definition for each origin, and the area of each figure represents the measured metrics. This is another difference between a Venn diagram and MAF.

Wettel et al. presented a 3D visualization approach, which gravitates around the city metaphor $[9,10]$. Their goal was to give the viewer a sense of locality to ease program comprehension. OC is inspired by Code City. Buildings or city-lots in Code City represent the context of software, such as packages and classes. The building height expresses its measured metrics. In contrast, buildings in OC represent the origins. Moreover, the building is composed of a color-coded stack, which denotes the functional layers.

There are many studies on the visualization of software. Caserta et al. surveyed $2 \mathrm{D}$ and $3 \mathrm{D}$ visualization techniques based on statistical aspects of software and its evolution [12]. Knight surveyed 3D visualization, and showed the important factors for visualization and how to use them [13]. 3D software visualization like a city has a decent history. Knight et al. also proposed Software World to create graphical abstractions of Java source code, which is one of the starting points for 3D software visualization [14]. Maletic et al. visualized UML class diagram in the form of layered $3 \mathrm{D}$ objects [15]. Similarly, OC is based on layered objects in 3D; however it is dedicated to visualization of origins. Panas et al. presented 3D City, which uses a city metaphor for software maintenance costs. As a result, their visualization technique expresses dangerous source code as an old or collapsed building [16]. Such methods can be applied to our techniques for additional expressions to represent the severity of the defects, etc. Although we implemented our tool as a web application, 
Fittkau et al. presented ExplorViz, which models live program traces with a webapplication utilizing WebGL $[17,18]$. Compared to our methods, which focus on the origin, their method provides a multi-level visualization from the landscape to the level of individual applications for system comprehension. Kobayashi et al. proposed SarF Map to visualize features and layers [19]. Langelier et al. proposed a metrics visualization technique named VERSO in which the graphical properties of buildings are height, color, and angle [20]. The idea of using colors to represent properties is similar to our approaches; if we need to represent more properties at one time, we could consider using the angle. Byelas et al. developed the area of interest, which expresses UML elements of shared properties by overlapping figures. Similar to MAF, the elements are colored like a contour diagram [21, 22]. Ducasse et al. proposed a Distribution map [7]. It visualizes a software system with colored rectangles, which express measured metrics of each file by group, like Java packages. Certainly, it is a more generic method than MAF. However, MAF can express whether an origin belongs to multiple groups and can visualize the ratio of measured metrics intuitively with overlapping figures. Girba et al. proposed the Ownership map, which visualizes the creation and modification history of a file with colored lines and circles [8]. It can express metrics, which our study calls origins by file. In contrast, MAF can express measured metrics simultaneously, but cannot express the origin in detail.

\section{Conclusions and Future Work}

We propose two visualization techniques: MAF and OC. These new methods are more efficient than primitive methods such as tables, bar charts, and pie charts. These methods must be used in conjunction with these primitive methods. Many developers have indicated that the proposed methods improve product quality. The result of the practical review process experiment shows the good effect quantitatively.

Our proposed techniques have the following advantages, disadvantages and limitations:

\section{- Advantages}

- MAF and OC are useful to grasp the value of the origins.

- MAF is useful to grasp the trend of a value.

- OC is useful to grasp the value by functional layers.

- Disadvantages

- MAF and OC are not self-sufficient and self-informative. They require additional information such as a table with numeric data.

- MAF should not be used when there are too many origins because hundreds or more colored regions may confuse users. 


\section{- Limitations}

- MAF and OC cannot be applied to projects that do not fit the concept of origins and functional layers.

In the future, we intend to investigate whether these methods can improve product quality by a continuous experiment. We also plan to refine our visualization tool. For example, our visualize application currently supports the function layer for the Android architecture only, but we would like to support additional architectures. This is our short-term goal. Additionally, we would like to create a tool that collects information about the management quality similar to a dashboard. We will implement a suggestion function, which should be useful for developer decision-making. Our long-term goal is to publish our tool as OSS.

\section{References}

1. R. Ishizue, H. Washizaki, Y. Fukazawa, S. Inoue, Y. Hanai, M. Kanazawa and K. Namba, Metrics visualization technique based on the origins and function layers for OSS-based development, in Proc. 2016 IEEE Working Conf. Software Visualization, 2016, pp. 71-75.

2. H.-M. Koo and I.-Y. Ko, An analysis of problem-solving patterns in open source software, Int. J. Softw. Eng. Knowl. Eng. 25(06) (2015) 1077-1103. [Online], Available: http:// www.worldscientific.com/doi/abs/10.1142/S0218194015500187.

3. Z. Wang, D. E. Perry and X. Xu, Characterizing individualized coding contributions of OSS developers from topic perspective, Int. J. Softw. Eng. Knowl. Eng. (2016) 1-34. [Online], Available: http://www.worldscientific.com/doi/abs/10.1142/S021819401750005X.

4. S. Sato, H. Washizaki, Y. Fukazawa, S. Inoue, H. Ono, Y. Hanai and M. Yamamoto, Effects of organizational changes on product metrics and defects, in Proc. 20th AsiaPacific Software Engineering Conf., Vol. 1, 2013, pp. 132-139.

5. F. Rohnert, R. Buschmann, H. Meunier and P. S. M. Stal, Pattern-oriented Software Architecture: A System of Patterns (Wiley Press, 1996).

6. Android interfaces and architecture, [Online]. Available: https://source.android.com/ devices/index.html [accessed 10 December 2016].

7. S. Ducasse, T. Gîrba and A. Kuhn, Distribution map, in Proc. 22nd IEEE Int. Conf. Software Maintenance, 2006, pp. 203-212.

8. T. Gîrba, A. Kuhn, M. Seeberger and S. Ducasse, How developers drive software evolution, in Proc. 8th Int. Workshop on Principles of Software Evolution, 2005, pp. 113-122.

9. R. Wettel and M. Lanza, Visualizing software systems as cities, in Proc. 4th IEEE Int. Workshop on Visualizing Software for Understanding and Analysis, 2007, pp. 92-99.

10. R. Wettel, M. Lanza and R. Robbes, Software systems as cities: A controlled experiment, in Proc. 33rd Int. Conf. Software Engineering, 2011, pp. 551-560. [Online]. Available: http://doi.acm.org/10.1145/1985793.1985868.

11. R. Ando, S. Sato, C. Uchida, H. Washizaki, Y. Fukazawa, S. Inoue, H. Ono, Y. Hanai, M. Kanazawa, K. Sone, K. Namba and M. Yamamoto, How does defect removal activity of developer vary with development experience? in Proc. 27th Int. Conf. Software Engineering and Knowledge Engineering, 2015, pp. 540-545.

12. P. Caserta and O. Zendra, Visualization of the static aspects of software: A survey, IEEE Trans. Vis. Comput. Graph. 17(7) (2011) 913-933.

13. C. Knight, System and software visualization, in Handbook of Software Engineering and Knowledge Engineering, Vol. 2 (2002), pp. 131-139. 
14. C. Knight and M. Munro, Virtual but visible software, in Proc. IEEE Int. Conf. Information Visualization, 2000, pp. 198-205.

15. J. I. Maletic, J. Leigh and A. Marcus, Visualizing software in an immersive virtual reality environment, in Proc. 23rd Int. Conf. Software Engineering Workshop on Software Visualization, Vol. 1, 2001, pp. 12-13.

16. T. Panas, R. Berrigan and J. Grundy, A 3d metaphor for software production visualization, in Proc. Seventh Int. Conf. Information Visualization, 2003, pp. 314-319.

17. F. Fittkau, J. Waller, C. Wulf and W. Hasselbring, Live trace visualization for comprehending large software landscapes: The explorviz approach, in Proc. 1st IEEE Working Conf. Software Visualization, 2013, pp. 1-4.

18. F. Fittkau, A. Krause and W. Hasselbring, Software landscape and application visualization for system comprehension with explorviz, Inf. Softw. Technol., 2016. [Online]. Available: //www.sciencedirect.com/science/article/pii/S0950584916301185.

19. K. Kobayashi, M. Kamimura, K. Yano, K. Kato and A. Matsuo, SARF map: Visualizing software architecture from feature and layer viewpoints, in Proc. 21st Int. Conf. Program Comprehension, 2013, pp. 43-52.

20. G. Langelier, H. Sahraoui and P. Poulin, Visualization-based analysis of quality for largescale software systems, in Proc. 20th IEEE/ACM Int. Conf. Automated Software Engineering, 2005, pp. 214-223. [Online]. Available: http://doi.acm.org/10.1145/ 1101908.1101941.

21. H. Byelas and A. Telea, Visualization of areas of interest in software architecture diagrams, in Proc. ACM Symp. Software Visualization, ser. SoftVis '06, 2006, pp. 105114. [Online]. Available: http://doi.acm.org/10.1145/1148493.1148509.

22. H. Byelas and A. Telea, Visualizing metrics on areas of interest in software architecture diagrams, in Proc. IEEE Pacific Visualization Symp., 2009, pp. 33-40. 